\title{
Red Blood Cells are Essential for Late Vasospasm Following Experimentally Induced Subarachnoid Hemorrhage in Dogs
}

\author{
Kazuhiko NOZAKI, Shinichiro OKAMOTO, Hiroji Yanamoto \\ and Haruhiko KIKUCHI
}

Department of Neurosurgery, Faculty of Medicine, Kyoto University, Kyoto

\begin{abstract}
The in vivo spasmogenic activity of various blood components was examined in dogs. Each blood fraction was injected into the cisterna magna at 0.5 or $1.0 \mathrm{ml} / \mathrm{kg}$ body weight, after the removal of $0.5 \mathrm{ml} / \mathrm{kg}$ body weight of cerebrospinal fluid, and vertebral angiography was then performed. Whole blood induced both early and late arterial spasm. Platelet-rich and platelet-poor plasma produced only early spasm, and no arterial narrowing was observed on days 1,3 , and 7 after injection. On the contrary, intracisternal injection of washed red blood cells $(0.5 \mathrm{ml} / \mathrm{kg}$ body weight) produced no arterial narrowing for 6 hours after injection and induced moderate arterial narrowing on days 1, 3, and 7 after injection. Hemolysate (a $10 \mathrm{gm} / \mathrm{dl}$ concentration of hemoglobin) produced prolonged monophasic arterial narrowing after injection. These results imply that red blood cells are required for late, prolonged arterial narrowing after experimental subarachnoid hemorrhage.
\end{abstract}

Key words: cerebral vasospasm, experimental subarachnoid hemorrhage, blood component, red blood cell, angiography

\section{Introduction}

The pathogenesis of cerebral vasospasm, which is often associated with subarachnoid hemorrhage (SAH), has not yet been elucidated, and many vasoactive substances are considered candidates as causative factors. Since erythrocyte breakdown products do not lose their contractile activity even after incubation, the role of erythrocytes and substances released from erythrocytes has been emphasized. $4,5,7,10,12,13.17-22)$ Cerebral vasospasm occurs several days after $\mathrm{SAH}$ and continues for 1 to 2 weeks. Taking into account the delayed onset of cerebral vasospasm, erythrocyte hemolysis seems likely to be involved. ${ }^{23)}$ A number of investigators have demonstrated the spasmogenicity of various blood components in vivo, ${ }^{2,12,14,24)}$ but the correlation between hemolysis and delayed onset of cerebral vasospasm has not yet been proved in vivo. Using an experimental SAH model, we attempted to determine which blood components are necessary for the

Received July 20, 1989; Accepted November 17, 1989 development of cerebral vasospasm.

\section{Materials and Methods}

Adult mongrel dogs of both sexes, weighing $7-13 \mathrm{~kg}$, were anesthetized with intramuscular injection of ketamine hydrochloride ( $5 \mathrm{mg} / \mathrm{kg}$ body weight) and intravenous infusion of sodium pentobarbital (20$30 \mathrm{mg} / \mathrm{kg}$ body weight). Spontaneous respiration was permitted via an endotracheal tube. Blood pressure, heart rate, and respiratory rate were monitored during the experiments. Arterial oxygen pressure $\left(\mathrm{PaO}_{2}\right)$, arterial carbon dioxide pressure $\left(\mathrm{PaCO}_{2}\right), \mathrm{pH}$, and base excess were also monitored.

Whole blood was obtained via a catheter inserted into the femoral artery and was drawn into nonheparinized tubes. Platelet-rich plasma was the supernatant obtained by centrifugation of heparinized arterial blood for 15 minutes at $100 \times G$ and $4^{\circ} \mathrm{C}$. Platelet-poor plasma was prepared as the supernatant of heparinized arterial blood obtained by centrifugation for 20 minutes at $2500 \times \mathrm{G}$ and $4^{\circ} \mathrm{C}$. The lower two-thirds of the sediment was washed three times with lactated Ringer's solution, then 
Table 1 Cell counts of each blood fraction

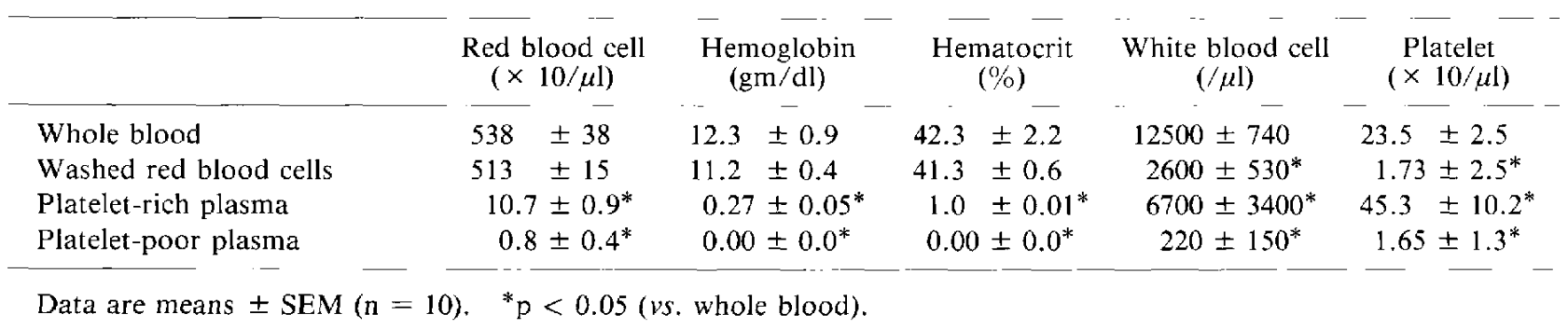

suspended in the same volume of the solution and used as washed red blood cells (RBCs). Hemolysate was the supernatant obtained by centrifugation of washed RBCs osmotically disrupted with distilled water $\left(10,000 \times \mathrm{G}, 20\right.$ minutes, $\left.4^{\circ} \mathrm{C}\right)$. The concentration of hemoglobin was adjusted to $10 \mathrm{gm} / \mathrm{dl}$ by the hemoglobin-cyanide method. The tubes used in the preparation of blood fractions were coated with polyvinyl alcohol to prevent the destruction of platelets. The cell counts of each blood fraction are shown in Table 1.

Each fraction was injected percutaneously into the cisterna magna through a 20 -gauge needle in a sterile and nontraumatic fashion. The animal was then kept in a head-down position for 30 minutes to facilitate settling of blood components around the basilar artery. In each case, $0.5 \mathrm{ml} / \mathrm{kg}$ body weight of cerebrospinal fluid (CSF) was removed prior to the intracisternal injection of blood components.

Vertebral angiography was performed following transfemoral catheterization under fluoroscopy. Angiographin $(3 \mathrm{ml})$ was injected rapidly by hand, and the projection was ventrodorsal at a fixed distance. Exposure was set at $60-80 \mathrm{kV}$ and $10-15 \mathrm{~mA}$. Angiograms were obtained before injection; 30, 60, and 120 minutes after injection, and 1,3, 7, 14, and 21 days after injection. The diameters of the basilar artery were measured at three sites on the angiograms, under optical magnification, before and after intracisternal injection of each blood component. The averages of the three pre- and three post-injection diameters were calculated, and the latter was expressed as a percentage of the former.

The three experiments conducted are outlined in Table 2. In experiment 1 , the spasmogenicity of each fraction was assessed by injecting $1.0 \mathrm{ml} / \mathrm{kg}$ body weight each of whole blood, washed RBCs, platelet-rich and platelet-poor plasma, hemolysate, and lactated Ringer's solution into the cisterna magna (hypervolumetric). In experiment 2 , the influence of increased intracranial pressure was eliminated by injection of only $0.5 \mathrm{ml} / \mathrm{kg}$ body weight each of whole blood, washed RBCs, platelet-rich
Table 2 Experimental designs

\begin{tabular}{|c|c|c|}
\hline & 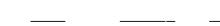 & \\
\hline & Dose & $\begin{array}{c}\text { No. of } \\
\text { dogs }\end{array}$ \\
\hline \multicolumn{3}{|c|}{ Experimental 1: hypervolumetric group } \\
\hline lactated Ringer's solution & $1 \mathrm{ml} / \mathrm{kg}$ & 3 \\
\hline platelet-rich plasma & $1 \mathrm{ml} / \mathrm{kg}$ & 5 \\
\hline platelet-poor plasma & $1 \mathrm{ml} / \mathrm{kg}$ & 5 \\
\hline washed red blood cells & $1 \mathrm{ml} / \mathrm{kg}$ & 5 \\
\hline $\begin{array}{l}\text { hemolysate } \\
(10 \mathrm{gm} / \mathrm{dl} \text { hemoglobin })\end{array}$ & $1 \mathrm{ml} / \mathrm{kg}$ & 5 \\
\hline whole blood & $1 \mathrm{ml} / \mathrm{kg}$ & 5 \\
\hline \multicolumn{3}{|c|}{ Experimental 2: isovolumetric group } \\
\hline lactated Ringer's solution & $0.5 \mathrm{ml} / \mathrm{kg}$ & 3 \\
\hline platelet-rich plasma & $0.5 \mathrm{ml} / \mathrm{kg}$ & 5 \\
\hline platelet-poor plasma & $0.5 \mathrm{ml} / \mathrm{kg}$ & 5 \\
\hline $\begin{array}{l}\text { hemolysate } \\
\text { (10 gm/dl hemoglobin })\end{array}$ & $0.5 \mathrm{ml} / \mathrm{kg}$ & 4 \\
\hline washed red blood cells & $0.5 \mathrm{ml} / \mathrm{kg}$ & 5 \\
\hline whole blood & $0.5 \mathrm{ml} / \mathrm{kg}$ & 5 \\
\hline \multicolumn{3}{|c|}{ Experimental 3: double injection group } \\
\hline platelet-rich plasma & $0.5 \mathrm{ml} / \mathrm{kg} \times 2$ & 5 \\
\hline whole blood & $0.5 \mathrm{ml} / \mathrm{kg} \times 2$ & 5 \\
\hline
\end{tabular}

and platelet-poor plasma, hemolysate, and lactated Ringer's solution into the cisterna magna (isovolumetric). In the third experiment, the effect of timing of injection was assessed by spacing of injections of whole blood and platelet-rich plasma $(0.5 \mathrm{ml} / \mathrm{kg}$ body weight) 48 hours apart.

Student's two-tailed t-test for paired samples was used to compare the percent diameter of the basilar artery before and after the injection of blood components. Differences with a p-value of less than 0.05 were considered statistically significant.

\section{Results}

The physiological parameters monitored during the experiments showed no significant changes.

Intracisternal injection of $0.5 \mathrm{ml} / \mathrm{kg}$ body weight of lactated Ringer's solution induced no significant 


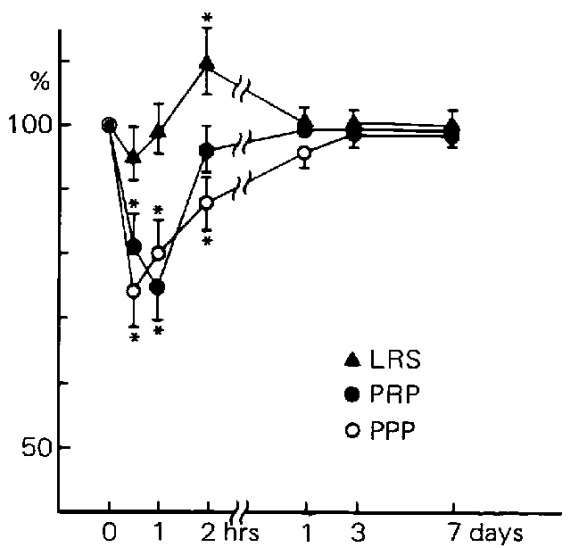

Fig. 1 Changes in the diameter of the basilar artery after intracisternal injection of $1.0 \mathrm{ml} / \mathrm{kg}$ body weight lactated Ringer's solution (LRS), platelet-rich plasma (PRP), and platelet-poor plasma (PPP). The diameter prior to injection is considered to be $100 \%$. The data are means. Vertical bars indicate two standard errors of the mean. ${ }^{*} \mathrm{p}<0.05$.

changes in the diameter of the basilar artery, whereas $1.0 \mathrm{ml} / \mathrm{kg}$ induced a slight decrease in the diameter 30 minutes after injection. This was followed by a slight increase in the arterial diameter 120 minutes after injection (Fig. 1). Intracisternal injection of $1.0 \mathrm{ml} / \mathrm{kg}$ body weight of both platelet-rich and platelet-poor plasma resulted in moderate contraction of the basilar artery 30-60 minutes after injection (Fig. 1). The artery gradually relaxed, and no significant contraction was observed on day 1 after injection. At $0.5 \mathrm{ml} / \mathrm{kg}$, both fractions induced the same pattern of contraction but to a milder degree.

Intracisternal injection of $1.0 \mathrm{ml} / \mathrm{kg}$ of washed RBCs produced biphasic contraction of the basilar artery (Fig. 2). The early contraction was most prominent 30 minutes after injection $(67 \pm 7.8 \%)$, and the late contraction on day 3 after injection $(67 \pm 6.0 \%)$. Contraction persisted for 2 to 3 weeks. On the other hand, $0.5 \mathrm{ml} / \mathrm{kg}$ of washed RBCs caused no significant change in the diameter of the basilar artery for up to 6 hours after injection (Fig. 2). However, moderate contraction was observed on day 1 (67 \pm $4.8 \%$ ) and disappeared on day 21 after injection (Fig. 3).

Intracisternal injection of hemolysate $(10 \mathrm{gm} / \mathrm{dl})$ induced prolonged, monophasic contraction of the basilar artery. At $0.5 \mathrm{ml} / \mathrm{kg}$ body weight, hemolysate induced moderate contraction immediately after injection (75 $\pm 5.6 \%$ ) (Fig. 4), which persisted until day 14 after injection. With injection of $1.0 \mathrm{ml} / \mathrm{kg}$ of hemolysate, exactly the same pattern and time

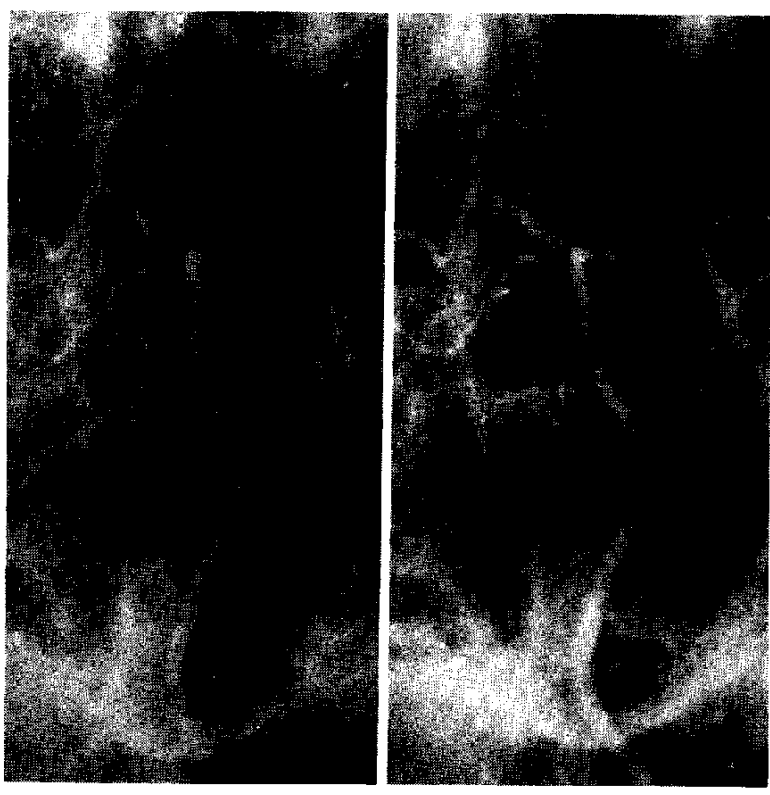

Fig. 2 Vertebral angiograms before (left) and 6 hours after (right) isovolumetric injection of washed red blood cells.

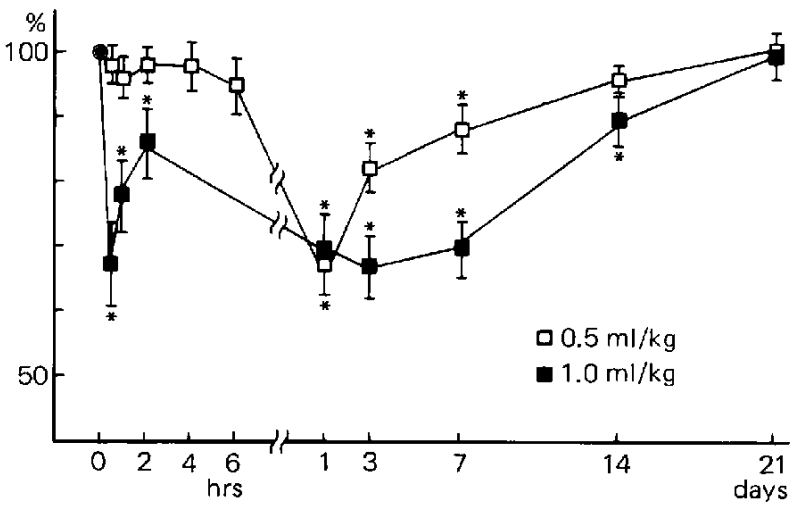

Fig. 3 Changes in the basilar artery diameter after intracisternal injection of 0.5 or $1.0 \mathrm{ml} / \mathrm{kg}$ body weight of washed red blood cells. ${ }^{*} \mathrm{p}<0.05$.

course were noted, although at this volume the contraction was marked $(63 \pm 7.5 \%)$ (Fig. 5 upper).

At $1.0 \mathrm{ml} / \mathrm{kg}$ body weight, whole blood produced biphasic arterial contraction. The early contraction was most marked 30 minutes after injection $(61 \pm$ $4.7 \%$ ), and the late contraction on day 3 after injection $(50 \pm 4.7 \%)$. The late contraction was more severe than that induced by washed RBCs and hemolysate. Injection of $0.5 \mathrm{ml} / \mathrm{kg}$ of whole blood resulted in the same pattern of biphasic contraction 

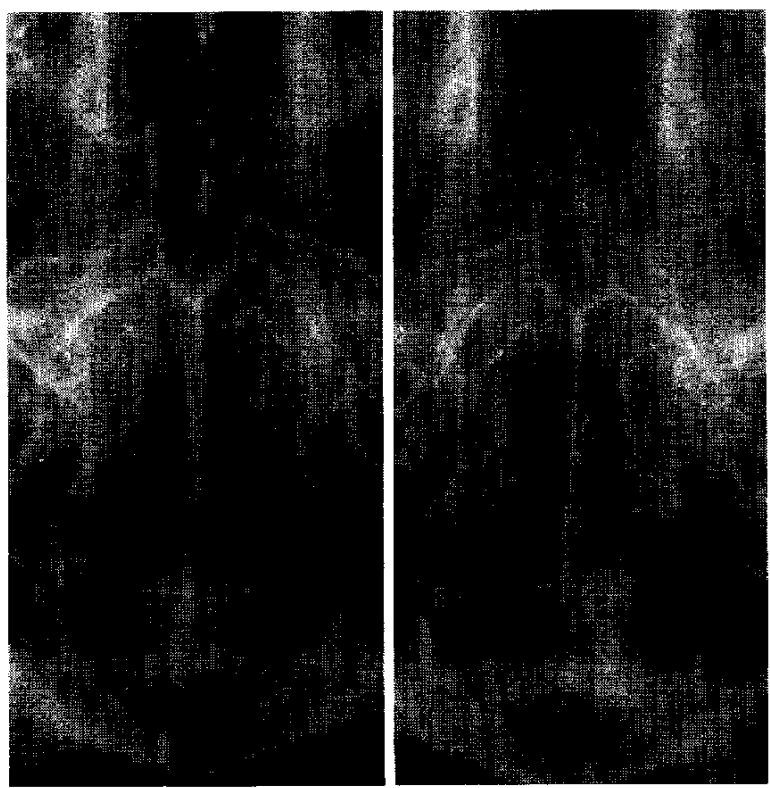

Fig. 4 Vertebral angiograms before (left) and 2 hours after (right) injection of $0.5 \mathrm{ml} / \mathrm{kg}$ body weight of hemolysate $(10 \mathrm{gm} / \mathrm{dl})$.

but to a lesser degree (Fig. 5 middle).

Two successive injections of whole blood $(0.5 \mathrm{ml} /$ $\mathrm{kg}$ body weight) 48 hours apart produced severe contraction of the basilar artery on day 7 after the first injection $(47 \pm 6.7 \%)$, and the contraction persisted through day 21 after the first injection (Fig. 5 middle). Platelet-rich plasma injected twice at a 48 -hour interval $(0.5 \mathrm{ml} / \mathrm{kg}$ body weight $)$ induced slight contraction of the basilar artery on day 3 after the first injection $(93 \pm 3.0 \%)$, but this contraction ceased by day 7 after the first injection (Fig. 5 lower). The contraction of the basilar artery was more severe after two injections of platelet-rich plasma than after one injection.

\section{Discussion}

Chronic cerebral vasospasm following the rupture of intracranial aneurysms has three striking features. First, its onset is delayed, occurring several days after the hemorrhage. Second, it persists for up to two weeks. Third, it is reversible, ceasing spontaneously after a certain period. However, the degree of vasoconstriction may be a factor in its reversibility, since severe vasospasm is usually fatal. The first two features imply that cerebral vasospasm is quite different from normal muscle contraction.

Various vasoconstrictive agents have been proposed as causes of cerebral vasospasm. Among
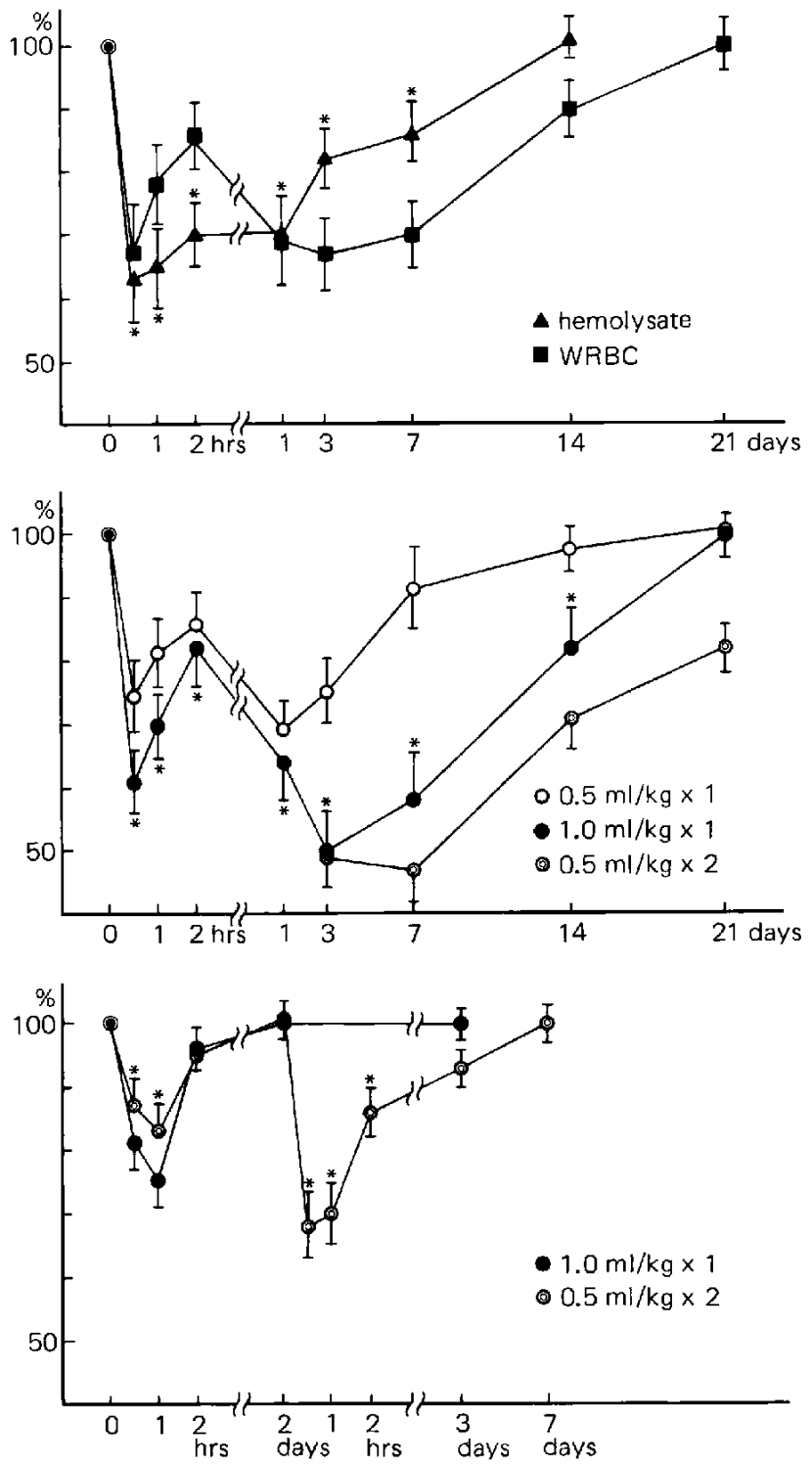

Fig. 5 Changes in the diameter of the basilar artery after intracisternal injections of $1.0 \mathrm{ml} / \mathrm{kg}$ body weight each of hemolysate $(10 \mathrm{gm} / \mathrm{dl}$ hemoglobin) and washed red blood cells (WRBC) (upper), after intracisternal injection of whole blood (middle), and after two intracisternal injections of 0.5 and $1.0 \mathrm{mg} / \mathrm{kg}$ body weight platelet-rich plasma 48 hours apart (lower). ${ }^{*} \mathrm{p}<0.05$.

these, certain erythrocyte breakdown products that may be released gradually during hemolysis are of particular interest in light of the time course of cerebral vasospasm. It has been shown that blood components other than erythrocytes lose their contractile activity after incubation for 24 hours at $37^{\circ} \mathrm{C}$ and that erythrocytes assume contractile 
activity only after incubation. ${ }^{17,187}$ It has also been reported that a blood-CSF mixture incubated for 5 to 10 days strongly induced contraction in isolated arterial strips or exposed cerebral arteries. ${ }^{8,15.20)}$ In this study, we demonstrated that only blood fractions containing erythrocytes were capable of inducing delayed and prolonged arterial narrowing in vivo. Boullin et al. ${ }^{2)}$ reported that intracisternally injected hemoglobin did not produce severe, chronic cerebral vasospasm in baboons. Other investigators ${ }^{11,1+1}$ successfully produced strong delayed arterial spasm in dogs by injecting hemoglobin intracisternally. In the present study, on the other hand, monophasic arterial spasm was observed after intracisternal injection of hemolysate. This discrepancy may be due to the species difference and/or a difference in the dosage of hemoglobin administered.

The degrees and time courses of prolonged arterial spasm induced by whole blood, washed RBCs, and hemolysate differed, whole blood being the most potent and hemolysate the weakest inducer. It is possible that there are differences in the clearance time of spasmogens from the subarachnoid space, and/or that some agent other than erythrocytes influences these events. Differences in the concentration of hemoglobin is a third possible explanation. Dense clots on the ventral surface of the brainstem and basal cistern were usually seen on day 3 after whole blood was injected into the cisterna magna, but were sometimes seen on day 3 after injection of washed RBCs. After intracisternal injection of hemolysate, only pigmentation was observed on the brain surface and arachnoid membrane. The concentrations of hemoglobin in the whole blood and washed RBCs were almost the same, while that of hemolysate was slightly lower. The difference in the degree of late spasm induced by whole blood and washed RBCs implies that other factors may contribute to the severity and persistence of late spasm.

It is generally accepted that early spasm is a transient contraction induced by certain vasoconstrictive agents, such as serotonin and $\mathrm{TXA}_{2}$, and by mechanical factors. ${ }^{1,3,16)}$ Intact RBCs per se have no vasoconstrictive activity in vitro; they become vasoactive only after hemolysis. ${ }^{18)}$ The present study shows that platelets and plasma participate in the genesis of early spasm, and that mechanical factors, such as an increase in intracranial pressure, are also involved, since the hypervolumetric injection of washed RBCs and lactated Ringer's solution $(1.0 \mathrm{ml} / \mathrm{kg}$ body weight) into the cisterna magna produced early arterial contraction. When an isovolumetric amount of washed RBCs was injected, only late spasm occurred. This suggests that early spasm is not necessary for the generation of late spasm, and that erythrocyte hemolysis may play an important role in the initiation of cerebral vasospasm.

In the clinical setting, the amount of blood in the subarachnoid space is well correlated with the frequency and degree of cerebral vasospasm. ${ }^{9}$ Zabramski et $a{ }^{25}{ }^{25}$ demonstrated that the volume and timing of blood injection is related to the degree and time course of late spasm in dogs. We found that the volume of platelet-rich plasma and whole blood was related to the severity of both early and late spasm. Also, neither a single injection nor two injections of platelet-rich plasma 48 hours apart induced late spasm in our study. DuBoulay et al. ${ }^{6)}$ reported, however, that platelets produced prolonged arterial spasm of the vertebral artery in monkeys. The species difference may account for this discrepancy.

In conclusion, we demonstrated in vivo that erythrocytes are necessary for the production of delayed, prolonged vasospasm and that platelets alone cannot induce late arterial contraction in dogs.

\section{Acknowledgment}

This paper was presented at the 47th Annual Meeting of the Japan Neurosurgical Society, held in Kobe on October 19-21, 1988.

\section{References}

1) Arutiunov AI, Baron MA, Majorova NA: The role of mechanical factors in the pathogenesis of shortterm and prolonged spasm of the cerebral arteries. $J$ Neurosurg 40: 459-472, 1974

2) Boullin DJ, Tagari P, DuBoulay GH, Aitken $V$, Hughes JT: The role of hemoglobin in the etiology of cerebral vasospasm. An in vivo study of baboons. $J$ Neurosurg 59: 231-236, 1983

3) Brawley BW, Strandness DE Jr, Kelly WA: The biphasic response of cerebral vasospasm in experimental subarachnoid hemorrhage. $J$ Neurosurg 28 : 1-8, 1968

4) Cheung ST, Mcllhany MP, Lim R, Mullan S: Preliminary characterization of vasocontractile activities in erythrocytes. J Neurosurg 53:37-43, 1980

5) Choku M: An experimental study of cerebral vasospasm, especially on spasmogenic factors in red blood cells. Osaka City Medical Journal 24: 211-222, 1975 (in Japanese)

6) DuBoulay GH, Hughes JT, Aitken V, Boullin DJ: Platelets in the subarachnoid space. A cause of acute and delayed arterial spasm, intimal damage and cerebral infarction. Acta Neurochir (Wien) 89: 6470,1987

7) Duff TA, Louie J, Feilbach JA, Scott G: Erythrocytes are essential for development of cerebral 
vasculopathy resulting from subarachnoid hemorrhage in cats. Stroke 19: 68-72, 1988

8) Endo S, Suzuki J: Experimental cerebral vasospasm after subarachnoid hemorrhage: Development and degree of vasospasm. Siroke 8: 702-707, 1977

9) Fisher CM, Kistler JP, Davis JM: Relation of cerebral vasospasm to subarachnoid hemorrhage visualized by computerized tomographic scanning. Neurosurgery 6: 1-9, 1980

10) Fujiwara S, Kuriyama $\mathrm{H}$ : Hemolysate-induced contraction in smooth muscle cells of the guinea pig basilar artery. Stroke 15: 503-510, 1984

11) Ishii $\mathrm{S}$, Nonaka $\mathrm{T}$ : Cerebral vasospasm in subarachnoid hemorrhage: With special reference to its mechanism. No To Shinkei 29: 829-840, 1977 (in Japanese)

12) Ito M: Experimental vasospasms. Significance of oxyhemoglobin, fibrin degradation products and breakdown products of white ghost in the pathogenesis of cerebral vasospasms. Treatment of vasospasms with gabexate mesilate and diphenhydramine. Neurol Med Chir (Tokyo) 20: 225-236, 1980 (in Japanese)

13) Lee TJ, Mcllhany F, Michael P, Sarwinski S: Erythrocyte extracts enhance neurogenic vasoconstriction of dog cerebral arteries in vitro. $J$ Cereb Blood Flow Metabol 4: 474-476, 1984

14) Mcllhany MP, Johns LM, Leipzig T, Patronas NJ, Brown FD, Mullan SF: In vivo characterization of vasocontractile activities in erythrocytes. $J$ Neurosurg 58: 356-361, 1983

15) Miyaoka $M$, Nonaka $T$, Watanabe $H$, Chigasaki $H$, Ishii $\mathrm{S}$ : Etiology and treatment of prolonged vasospasm. Experimental and clinical studies. Neurol Med Chir (Tokyo) 16: 103-114, 1976 (in Japanese)

16) Nagai H, Suzuki Y, Sugiura $M$, Noda S, Mabe H: Experimental cerebral vasospasm. Part 1: Factors contributing to early spasm. J Neurosurg 41: 285
292,1974

17) Okwuasaba F, Cook D, Weir B: Changes in vasoactive properties of blood products with time and attempted identification of the spasmogens. Stroke 12: $775-780,1981$

18) Osaka K: Prolonged vasospasm produced by the breakdown products erythrocytes. $J$ Neurosurg 47 : 403-411, 1977

19) Ozaki N, Mullan S: Possible role of the erythrocyte in causing prolonged cerebral vasospasm. I Neurosurg 51: 773-778, 1979

20) Sonobe M, Suzuki J: Vasospasmogenic substance produced following subarachnoid haemorrhage, and its fate. Acta Neurochir (Wien) 44: 97-106, 1978

21) Tanishima T: Cerebral vasospasm: Contractile activity of hemoglobin in isolated canine basilar arteries. $J$ Neurosurg 53: 787-793, 1980

22) Toda N, Shimizu K, Ohta T: Mechanism of cerebral arterial contraction induced by blood constituents. $J$ Neurosurg 53: 312-322, 1980

23) Tourtellotte WW, Metz LN, Bryan ER, DeJong RN: Spontaneous subarachnoid hemorrhage: Factors affecting the rate of clearing of the cerebrospinal fluid. Neurology (Minneap) 14: 301-306, 1964

24) White RP, Hagen AA, Morgan H, Dawson WN, Robertson JT: Experimental study on the genesis of cerebral vasospasm. Stroke 6: 52-57, 1975

25) Zabramski JM, Spetzler RF, Bonstelle C: Chronic cerebral vasospasm: Effect of volume and timing of hemorrhage in a canine model. Neurosurgery 18: 16,1986

Address reprint requests to: K. Nozaki, M.D., Department of Neurosurgery, Faculty of Medicine, Kyoto University, 54 Kawahara-cho, Shogoin, Sakyo-ku, Kyoto 606, Japan. 